\title{
Propiedades psicométricas de la escala de salud mental positiva en jóvenes colombianos: un estudio exploratorio
}

\author{
Psychometric Properties of the Positive Mental Health Scale with \\ Colombian Young People: An Exploratory Study
}

\author{
Andrés Gómez-Acosta 1 ORCID , Stéfano Vinaccia-Alpi ${ }^{2} \underline{\mathrm{ORCID}}$, Willian Sierra-Barón ${ }^{3} \mathrm{ORCID}$ \\ 1 Fundación Universitaria Sanitas \\ 2 Universidad del Sinú \\ 3 Universidad SurColombiana
}

Colombia

Fecha correspondencia:

Recibido: mayo 7 de 2019.

Aceptado: abril 7 de 2020.

Forma de citar:

Gómez-Acosta, A., Vinaccia-Alpi,

S., \& Sierra-Barón, W. (2020).

Propiedades psicométricas de la

Escala de Salud Mental Positiva en

jóvenes colombianos: un estudio

exploratorio. Rev. CES PSico, 13(2),

102-112.

Open access

(c) Copyright

Licencia creative commons

Etica de publicaciones

Revisión por pares

Gestión por Open Journal System

DOI: http://dx.doi.org/10.21615/

cesp.13.2.7

ISSN: 2011-3080

Comparte

\section{Resumen}

Antecedentes: Si bien se han desarrollado investigaciones previas que examinan las propiedades psicométricas de la Escala de Salud Mental Positiva original de Lluch en otros países latinoamericanos, hasta el momento no se ha documentado un estudio similar en Colombia. Objetivo: Revisar las propiedades psicométricas de la Escala de Salud Mental Positiva en jóvenes colombianos. Método: Estudio de tipo instrumental - psicométrico, con una muestra de 886 personas ( $63.4 \%$ mujeres) entre 13 y 25 años de edad. Resultados: El análisis factorial muestra una correspondencia con los seis factores propuestos en la versión original de la prueba, con una varianza explicada del 42,90\%; así como indicadores aceptables de confiabilidad por cada factor y de correlaciones entre ítems. Conclusiones: Estos hallazgos aportan evidencia empírica a favor del uso de la Escala de Salud Mental Positiva con jóvenes, no obstante, es necesario un ejercicio posterior de validación que aporte indicadores psicométricos más robustos.

Palabras clave: salud mental positiva; propiedades psicométricas; población joven.

\section{Abstract}

Background: While previous research examining the psychometric properties of Lluch's original Positive Mental Health Scale has been conducted in other Latin American countries, a similar research in Colombia has not been documented to date. Objective: To review the psychometric properties of the Positive Mental Health Scale in Colombian young people. Method: Instrumental - psychometric study, with a sample of 886 people ( $63.4 \%$ women) between 13 and 25 years old. Results: The factorial analysis [FA] shows a correspondence with the six factors proposed in the original version of the test, with an explained variance of $42.90 \%$, as well as acceptable indicators of reliability for each factor and correlations between items. Conclusions: These findings provide empirical evidence in favor of the use of the Positive 


\section{Sobre los autores:}

1. Doctorando en Psicología, Magíster en Psicología y Psicólogo. Líder del grupo de investigación Psychology and Health Sanitas, Fundación Universitaria Sanitas, Bogotá D.C., Colombia.

2. PhD. en Psicología Clínica y de la Salud. Especialista en Medicina Psicosomática. Psicólogo. Grupo I FLOR, Universidad del Sinú, Montería, Colombia.

3. Doctorando en Psicología. Magíster en Educación y Psicólogo. Líder del grupo de investigación Desarrollo Humano y Sostenibilidad Ambiental, Universidad SurColombiana, Neiva, Colombia.
Mental Health Scale with young people; however, a subsequent validation exercise is needed to provide more robust psychometric indicators.

Keywords: positive mental health; psychometric properties; young people.

\section{Introducción}

La psicología de la salud contemporánea propone una alternativa conceptual distinta al abordaje patogénico clásico (Salanova, 2008), considerando los estados psicológicos positivos como factores protectores de la salud física y mental (Seligman \& Csikszentmihalyi, 2000). Esta perspectiva denominada "salud mental positiva [SMP]", es concebida como un estado de bienestar en el cual el individuo identifica sus propias aptitudes, afronta las presiones diarias de la vida, es productivo y contribuye efectivamente a la comunidad (Organización Mundial de la Salud [OMS], 2018).

En ese orden, la consideración de las potencialidades de los sujetos desde una postura de SMP se constituye en una fuente importante de recursos para el desempeño adecuado en situaciones cotidianas estresantes, el diseño de programas de prevención e intervención, el establecimiento de planes de recuperación y, en general, la adquisición y mantenimiento de competencias que conducen a un desarrollo personal exitoso (Vásquez, Hervas, Rahona, \& Gómez, 2009).

Teniendo en cuenta la importancia del concepto SMP en el ámbito de la salud, se han realizado estudios para identificar su presencia en niños trabajadores (Amar et al., 2008), personas con discapacidad física (González, Astudillo, \& Hurtado, 2012), estudiantes universitarios (Medina, Serrano, Martínez, \& Cardona, 2013) y equipos de salud mental (Vidal, Rivas, \& Bustos, 2015). La SMP se ha relacionado con reducción de la predisposición a enfermedades mentales (Keyes, Dhingra, \& Simoes, 2010), optimismo disposicional y adaptación académica (Londoño, 2009), competencias comunicativas en profesores (Orellana et al., 2012), fortalecimiento de la religiosidad y espiritualidad (Ganga \& Kutty, 2013), resiliencia (Srivastava, 2011), posición socioeconómica (Gilmour. 2014), consumo responsable de alcohol y comportamiento no violento (Gómez-Acosta \& Londoño, 2013) y autocuidado en salud (Gómez-Acosta, 2017).

Si bien se han realizado esfuerzos para medir la SMP (Vaillant, 2012), se observa que en diversos casos se han evaluado conceptos traslapados como emociones positivas, bienestar subjetivo e inteligencia emocional (Muñoz, Restrepo, \& Cardona, 2016). En ese orden, la medición del constructo SMP es aún incipiente, no obstante, se destaca el trabajo de Lluch (1999) quien, además de crear una escala útil para su evaluación, formuló un modelo que permitió operacionalizar la propuesta de SMP original de Jahoda (1958) a partir de seis dimensiones (satisfacción personal, actitud prosocial, autocontrol, autonomía, solución de problemas - autoactualización y habilidades de relación interpersonal), que pueden variar de acuerdo con criterios como la edad, el nivel académico y el sexo (Gilmour, 2014; Keyes et al,. 2010).

La Escala de Salud Mental Positiva de Lluch en su momento se desarrolló con estudiantes universitarios de una misma carrera (enfermería), y para confirmar su nivel predictivo ha sido replicada con poblaciones más heterogéneas (Lluch, 2002). Así, por ejemplo, se han realizado estudios para examinar sus propiedades psicométricas y validar el instrumento en México, con adultos obteniendo un alfa $=0.97$ (Barrera \& Flores, 2013), con trabajadores obteniendo un alfa $=0.86$, (Martínez et al., $\underline{2015}$ ) y con niños obteniendo un alfa = 0.80 (González \& Valdéz, 2016); en España, 
con profesores universitarios, en el que se obtuvo un alfa $=0.88$, (Hurtado et al., 2018) y en Portugal, con estudiantes universitarios, en el que se obtuvo un alfa = 0.92. (Sequiera et al., 2014).

En Colombia no existen estudios de adaptación, validación o exploración de las propiedades psicométricas de instrumentos que midan constructos desde la perspectiva paradigmática de la psicología positiva; y los que han sido adaptados parten de la lógica patogénica de identificación y evaluación centrada en el riesgo y la vulnerabilidad (Fernández-Rios \& Vilarino, 2018). Además, algunos instrumentos que evalúan la dimensión psicológica del proceso salud - enfermedad que se han creado o adaptado en Colombia presentan problemas para su acceso y disponibilidad, y se sustentan en estudios realizados con muestras limitadas (Londoño \& Alejo, 2017). Dado lo anterior, la presente investigación se plantea como objetivo revisar las propiedades psicométricas de la Escala de Salud Mental Positiva en una muestra amplia de jóvenes colombianos.

\section{Método}

\section{Diseño}

Corresponde a una investigación empírico - analítica, psicométrica y exploratoria, en la medida en que busca identificar si un instrumento (Escala de Salud Mental Positiva) puede medir el constructo (SMP) en una población determinada (jóvenes colombianos).

\section{Participantes}

Se obtuvo una muestra estratificada, a conveniencia de 886 jóvenes 324 hombres y 562 mujeres), 406 provenientes de ocho instituciones educativas de secundaria con edades comprendidas entre 13 y 25 años $(X=15.45$, d.e. $=1.39)$, y 460 de diferentes carreras de pregrado de cinco universidades, con edades comprendidas entre 16 y 25 años ( $X=19.87$, d.e. $=2.55$ ). El 49.2\% del total de la muestra vivía en estratos socioeconómico bajo, y el porcentaje restante pertenecía a estratos medios.

\section{Instrumento}

La Escala de Salud Mental Positiva, diseñada por Lluch (1999), mide el nivel de salud positiva de las personas, y engloba aspectos como: Satisfacción Personal (F1), Actitud Prosocial (F2), Autocontrol (F3), Autonomía (F4), Resolución de Problemas y Autoactualización (F5) y Habilidades de Relación Interpersonal (F6). Consta de 39 ítems distribuidos en los seis factores antes mencionados, cuyas opciones de respuesta se presentaron en una escala tipo Likert de cuatro alternativas, que va desde muy frecuente hasta nunca. La calificación está dada por la suma de los puntajes obtenidos en cada factor y una suma total de los mismos. Amar et al. (2008) determinaron un índice de confiabilidad del 0.66 en población de menores trabajadores de la costa atlántica colombiana y Londoño (2009) de 0.87 en estudiantes universitarios del mismo país.

\section{Procedimiento}

Inicialmente se solicitaron las respectivas autorizaciones en los centros educativos (colegios y universidades), para proceder a la socialización del estudio a los participantes y la obtención de los correspondientes avales (firmas de consentimientos y asentimientos informados), conforme a las consideraciones éticas establecidas para la investigación con sujetos humanos en Colombia (Ministerio de Salud, 1993; Congreso de Colombia, 2006). 
La aplicación del instrumento se realizó en las instalaciones de las instituciones educativas, en horario habitual, y conjuntamente se obtuvieron los datos sociodemográficos mediante una ficha de registro. Esta labor la adelantaron auxiliares de investigación, estudiantes de últimos semestres de psicología, entrenados tanto en aplicación de instrumentos psicométricos como en la resolución de las posibles dudas referidas por los participantes. El tiempo promedio de la aplicación fue de 16 minutos con los estudiantes de secundaria, y de 13 minutos con los universitarios. Se atendieron las consultas de los respondientes y se verificó que las hojas de respuesta fueran diligenciadas completamente para reducir la perdida de datos y la inconsistencia en los mismos.

No se consideró una revisión previa de los ítems de la Escala de Salud Mental Positiva o un pilotaje previo, dado que dicho instrumento ha sido previamente utilizado en Colombia con diferentes muestras (Amar et al., 2008; Gómez-Acosta \& Londoño, 2013; Londoño, 2009; Medina et al., 2012) sin que se hayan reportado dificultades en la comprensión de los ítems por parte de los participantes.

Finalmente, se brindó un informe general de los hallazgos y uno particularizado para cada institución, y se brindaron recomendaciones para el uso de la información y el fortalecimiento de la SMP en la población estudiada.

\section{Análisis de datos}

Los datos obtenidos se analizaron con el software SPSS $24{ }^{\circledR}$; se realizó un análisis factorial con extracción de análisis de componentes principales y rotación Varimax. Se establecieron los índices de confiabilidad con Alfa de Cronbach y se revisó la consistencia interna a partir de una matriz de correlaciones.

\section{Resultados}

La prueba Kolmogorov-Smirnov permitió identificar que la distribución de los factores de la Escala de SMP correspondió a los preceptos de normalidad. Adicionalmente, y con el fin de observar cómo se comportan los factores de la Escala SMP en la muestra, se analizaron las diferencias de medianas por sexo, nivel educativo, estrato socioeconómico y por rango de edad, y se observaron diferencias estadísticamente significativas en todos los factores excepto Autocontrol, tal como se observa en la Tabla 2.

Tabla 1. Estadísticos descriptivos de los factores de la Escala de SMP

\begin{tabular}{|c|c|c|c|c|c|c|c|c|}
\hline \multirow{2}{*}{$\begin{array}{c}\text { Factores de la Escala } \\
\text { de SMP }\end{array}$} & \multirow[b]{2}{*}{$\min$} & \multirow[b]{2}{*}{$\max$} & \multirow[b]{2}{*}{ media } & \multirow[b]{2}{*}{ d.s. } & \multicolumn{4}{|c|}{ Diferencia de medias } \\
\hline & & & & & Sexo & $\begin{array}{c}\text { Nivel } \\
\text { educativo }\end{array}$ & Estrato & $\begin{array}{l}\text { Rango } \\
\text { de Edad }\end{array}$ \\
\hline Satisfacción general & 1.0 & 4.0 & 3.31 & 0.52 & $0.00^{*}$ & $0.00^{*}$ & & $0.01^{*}$ \\
\hline Actitud prosocial & 1.0 & 4.0 & 3.21 & 0.51 & $0.00^{*}$ & $0.00^{*}$ & & $0.00^{*}$ \\
\hline Autocontrol & 1.0 & 4.0 & 2.78 & 0.56 & & & & \\
\hline Autonomía & 1.0 & 4.0 & 3.08 & 0.58 & & $0.00^{*}$ & & \\
\hline Solución de problemas & 1.0 & 4.0 & 3.03 & 0.52 & & $0.00^{*}$ & & $0.00^{*}$ \\
\hline Habilidades sociales & 1.0 & 4.0 & 3.05 & 0.49 & $0.00^{*}$ & $0.00^{*}$ & $0.04^{*}$ & $0.00^{*}$ \\
\hline SMP Total & 1.0 & 4.0 & 3.07 & 0.53 & $0.00^{*}$ & $0.00^{*}$ & & $0.00^{*}$ \\
\hline
\end{tabular}

* Significación estadística menor al 0.05 
Para determinar si el tamaño muestral era suficiente para proceder con el análisis factorial exploratorio, se calcularon los correspondientes coeficientes Kaiser-Meyer-Olkin y prueba de esfericidad de Bartlett, los cuáles se presentan en la Tabla 3. De acuerdo con dichos indicadores, se evidenció que la muestra es suficiente para el desarrollo del análisis factorial.

Tabla 2. Prueba de adecuación y suficiencia muestral

\begin{tabular}{llr}
\hline \multicolumn{2}{l}{ Medida Kaiser-Meyer-Olkin de adecuación de muestreo } & $\mathbf{0 . 9 0}$ \\
\hline \multirow{3}{*}{ Prueba de esfericidad de Bartlett } & Aprox. Chi-cuadrado & 9595.89 \\
\cline { 2 - 3 } & gl & 741.00 \\
\cline { 2 - 3 } & Sig. & $0.00^{*}$ \\
\hline * Significación estadística menor al 0.05 & &
\end{tabular}

Tal como se observa en la Tabla 3, se presentaron correlaciones positivas y superiores a 0.25 entre todos los ítems y el total de la prueba. De igual forma, los alfas de Cronbach se mantienen estables si se elimina algún reactivo de la Escala de SMP, por lo que se entiende que la prueba en su totalidad es confiable. Asimismo, al llevar a cabo el procedimiento de Análisis Factorial Exploratorio con el método de componentes principales y rotación Varimax, se encuentra que la prueba conserva la estructura factorial de la escala original.

Tabla 3. Matriz de carga factorial de cada reactivo, extracción, correlaciones ítem - prueba y alfa de Cronbach si se suprime el elemento de la Escala de SMP

\begin{tabular}{|c|c|c|c|c|c|c|c|c|c|c|}
\hline Ítems & F1 & $F 2$ & F3 & $F 4$ & F5 & F6 & $\begin{array}{c}\% \\
\text { Varianza } \\
\text { aportado }\end{array}$ & Extracción & $\begin{array}{l}\text { Correlación } \\
\text { total de } \\
\text { elementos } \\
\text { corregida }\end{array}$ & $\begin{array}{l}\text { Alfa de } \\
\text { Cronbach si } \\
\text { el ítem se } \\
\text { suprime }\end{array}$ \\
\hline ISMP 17 & 0.59 & & & & & & 1.93 & 0.51 & 0.52 & 0.88 \\
\hline ISMP 36 & 0.57 & & & & & & 1.05 & 0.45 & 0.45 & 0.88 \\
\hline ISMP 27 & 0.57 & & & & & & 1.47 & 0.52 & 0.48 & 0.88 \\
\hline ISMP 28 & 0.54 & & & & & & 1.43 & 0.61 & 0.40 & 0.88 \\
\hline ISMP 32 & 0.54 & & & & & & 1.29 & 0.41 & 0.4 & 0.88 \\
\hline ISMP 23 & 0.53 & & & & & & 1.69 & 0.37 & 0.4 & 0.88 \\
\hline ISMP 37 & 0.52 & & & & & & 1.05 & 0.49 & 0.38 & 0.88 \\
\hline ISMP 16 & 0.52 & & & & & & 2.00 & 0.42 & 0.50 & 0.88 \\
\hline ISMP 25 & 0.50 & & & & & & 1.60 & 0.43 & 0.34 & 0.88 \\
\hline ISMP 20 & 0.50 & & & & & & 1.85 & 0.47 & 0.42 & 0.88 \\
\hline ISMP 29 & 0.50 & & & & & & 1.41 & 0.5 & 0.29 & 0.88 \\
\hline ISMP 15 & 0.47 & & & & & & 2.01 & 0.47 & 0.47 & 0.88 \\
\hline ISMP 18 & 0.36 & & & & & & 1.92 & 0.42 & 0.37 & 0.88 \\
\hline ISMP 39 & & 0.65 & & & & & 0.93 & 0.6 & 0.40 & 0.88 \\
\hline
\end{tabular}




\begin{tabular}{|c|c|c|c|c|c|c|c|c|c|c|}
\hline Ítems & $F 1$ & $F 2$ & F3 & $F 4$ & F5 & F6 & $\begin{array}{c}\% \\
\text { Varianza } \\
\text { aportado }\end{array}$ & Extracción & $\begin{array}{c}\text { Correlación } \\
\text { total de } \\
\text { elementos } \\
\text { corregida }\end{array}$ & $\begin{array}{c}\text { Alfa de } \\
\text { Cronbach si } \\
\text { el ítem se } \\
\text { suprime }\end{array}$ \\
\hline ISMP 38 & & 0.64 & & & & & 1.01 & 0.68 & 0.39 & 0.88 \\
\hline ISMP 31 & & 0.52 & & & & & 1.35 & 0.52 & 0.45 & 0.88 \\
\hline ISMP 14 & & 0.49 & & & & & 2.21 & 0.50 & 0.49 & 0.88 \\
\hline ISMP 7 & & 0.49 & & & & & 2.79 & 0.44 & 0.47 & 0.88 \\
\hline ISMP 4 & & 0.48 & & & & & 3.80 & 0.46 & 0.36 & 0.88 \\
\hline ISMP 12 & & 0.35 & & & & & 2.30 & 0.49 & 0.40 & 0.88 \\
\hline ISMP 9 & & & 0.65 & & & & 2.59 & 0.55 & 0.43 & 0.88 \\
\hline ISMP 8 & & & 0.63 & & & & 2.71 & 0.51 & 0.41 & 0.88 \\
\hline ISMP 1 & & & 0.48 & & & & 19.78 & 0.42 & 0.35 & 0.88 \\
\hline ISMP 30 & & & 0.47 & & & & 1.36 & 0.35 & 0.33 & 0.88 \\
\hline ISMP 10 & & & & 0.66 & & & 2.47 & 0.59 & 0.37 & 0.88 \\
\hline ISMP 19 & & & & 0.60 & & & 1.88 & 0.5 & 0.35 & 0.88 \\
\hline ISMP 13 & & & & 0.60 & & & 2.21 & 0.49 & 0.31 & 0.88 \\
\hline ISMP 35 & & & & 0.46 & & & 1.56 & 0.63 & 0.29 & 0.88 \\
\hline ISMP 34 & & & & 0.37 & & & 1.19 & 0.45 & 0.33 & 0.88 \\
\hline ISMP 21 & & & & & 0.69 & & 1.73 & 0.62 & 0.41 & 0.88 \\
\hline ISMP 22 & & & & & 0.69 & & 1.70 & 0.59 & 0.38 & 0.88 \\
\hline ISMP 5 & & & & & 0.68 & & 3.43 & 0.55 & 0.3 & 0.88 \\
\hline ISMP 26 & & & & & 0.56 & & 1.52 & 0.50 & 0.3 & 0.88 \\
\hline ISMP 2 & & & & & 0.32 & & 7.89 & 0.43 & 0.36 & 0.88 \\
\hline ISMP 6 & & & & & 0.31 & & 2.90 & 0.52 & 0.32 & 0.88 \\
\hline ISMP 24 & & & & & & 0.59 & 1.63 & 0.56 & 0.36 & 0.88 \\
\hline ISMP 11 & & & & & & 0.50 & 2.38 & 0.55 & 0.25 & 0.88 \\
\hline ISMP 3 & & & & & & 0.49 & 5.11 & 0.46 & 0.34 & 0.88 \\
\hline ISMP 33 & & & & & & 0.39 & 1.22 & 0.51 & 0.39 & 0.88 \\
\hline
\end{tabular}

Método de extracción: análisis de componentes principales.

Método de rotación: Varimax con normalización Kaiser.

Adicionalmente, tal como se observa en el gráfico de sedimentación correspondiente (ver Figura 1), se corrobora la distribución de los ítems en los factores.

El cuestionario abreviado explica más varianza tanto en el total como en cada uno de los factores, aun cuando se redistribuyen los ítems en los factores, y los porcentajes de varianza explicada por los mismos (ver Tabla 4). 


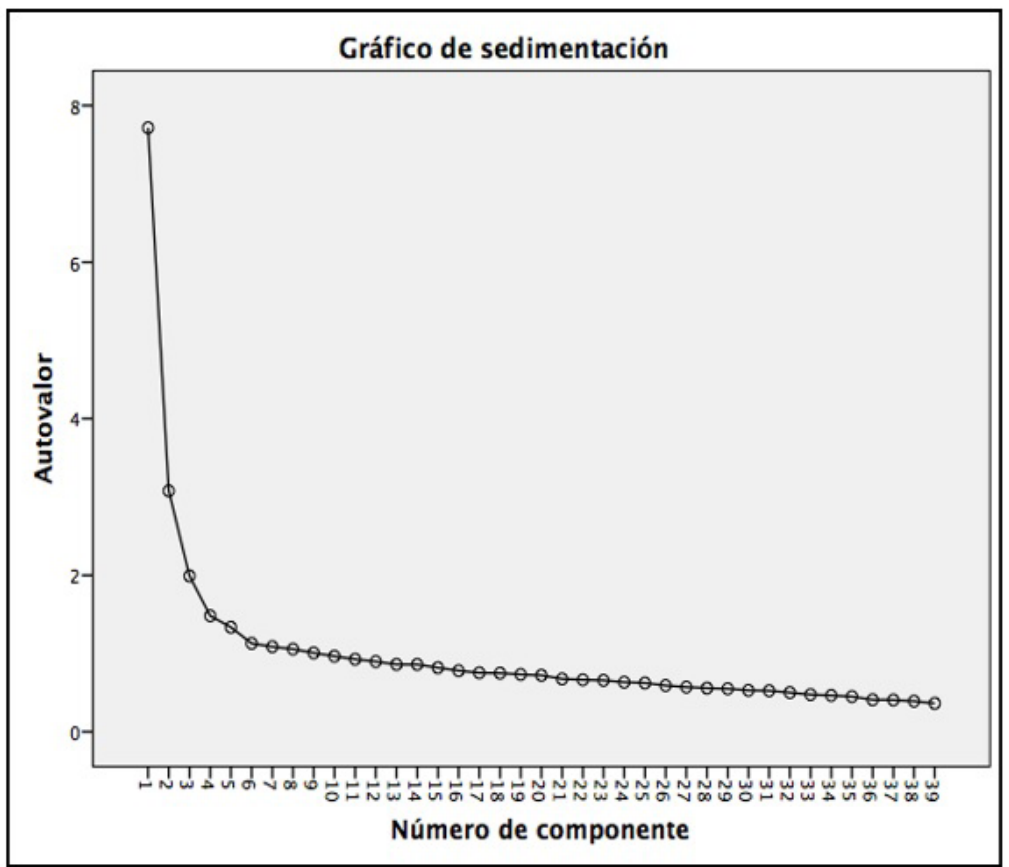

Figura 1. Gráfico de sedimentación de la aplicación en jóvenes colombianos de la Escala de SMP.

Tabla 4. Comparativo de las propiedades psicométricas de la Escala de SMP

\begin{tabular}{lcccccc}
\hline & \multicolumn{2}{c}{ Versión Final de Lluch (1999) } & \multicolumn{3}{c}{$\begin{array}{c}\text { Aplicación en jóvenes } \\
\text { Factombianos }\end{array}$} \\
\cline { 2 - 7 } \begin{tabular}{l} 
SMP \\
\cline { 2 - 7 }
\end{tabular} & $\begin{array}{c}\boldsymbol{N}^{\circ} \\
\text { İtems de }\end{array}$ & $\begin{array}{c}\text { \% de } \\
\text { varianza } \\
\text { explicada }\end{array}$ & $\begin{array}{c}\text { Alpha de } \\
\text { Cronbach }\end{array}$ & $\begin{array}{c}\mathbf{N}^{\circ} \\
\text { Ítems }\end{array}$ & $\begin{array}{c}\text { \% de } \\
\text { varianza } \\
\text { explicada }\end{array}$ & $\begin{array}{c}\text { Alpha de } \\
\text { Cronbach }\end{array}$ \\
\hline F1. Satisfacción general & 8 & 23.10 & 0.82 & 13 & 11.35 & 0.81 \\
\hline F2. Actitud prosocial & 5 & 6.70 & 0.58 & 7 & 7.37 & 0.74 \\
\hline F3. Autocontrol & 5 & 4.90 & 0.81 & 4 & 6.57 & 0.59 \\
\hline F4. Autonomía & 5 & 4.40 & 0.77 & 5 & 6.53 & 0.60 \\
\hline F5. Sol. Problemas-Autoact. & 9 & 3.90 & 0.79 & 6 & 6.50 & 0.67 \\
\hline F6. Habilidades sociales & 7 & 3.80 & 0.71 & 4 & 4.56 & 0.51 \\
\hline Cuestionario SMP & 39 & 46.80 & 0.90 & 39 & 42.90 & 0.88 \\
\hline
\end{tabular}

Finalmente, se desarrolló un análisis de Guttman para contrastar la consistencia interna de la escala total, obteniendo un indicador aceptable de 0.86 , por lo cual se reconoce alta correlación y poca variabilidad entre los puntajes de cada una de las mitades asignadas aleatoriamente.

\section{Discusión}

La presente investigación tuvo como objetivo revisar las propiedades psicométricas de la Escala de Salud Mental Positiva (SMP) en una muestra de jóvenes colombianos, dada la ausencia de estudios preliminares de su validez y confiabilidad en el contexto colombiano. 
Pág 109

Los resultados encontrados mediante el análisis factorial con extracción de análisis de componentes principales y rotación Varimax, agrupan los reactivos de la Escala de Salud Mental Positiva (SMP) en una estructura de seis factores, tal como en la escala original de Llunch, (1991) y con un porcentaje aceptable de varianza explicada de 42.90 en la escala total. Por otro lado, los análisis psicométricos de la escala total de SMP arrojaron una consistencia interna alta (alfa de Cronbach =.88), cercana a la obtenida por Sequeira et al. (2014) con estudiantes universtarios portugueses.
Los resultados encontrados mediante el análisis factorial con extracción de análisis de componentes principales y rotación Varimax, agrupan los reactivos de la Escala de SMP en una estructura de seis factores, tal como en la escala original de Llunch. (1991) y con un porcentaje aceptable de varianza explicada de 42.90 en la escala total. Por otro lado, los análisis psicométricos de la escala total de SMP arrojaron una consistencia interna alta (alfa de Cronbach =.88), cercana a la obtenida por Sequeira et al. (2014) con estudiantes universtarios portugueses. Con relación a los factores de la Escala de SMP que obtuvieron mayor confiabilidad fueron: Satisfacción general (alfa de Cronbach $=.81$ ) y Actitud prosocial (alfa de Cronbach $=.74$ ) mientras los factores Solución de problemas (alfa de Cronbach =.67), Autocontrol (alfa de Cronbach =.59), Autonomía (alfa de Cronbach $=.60$ ) y Solución de problemas (=.51) presentaron una consistencia interna más baja.

Llama la atención que en el presente estudio el factor Actitud Prosocial obtuvo una puntuación mucho más alta del coeficiente alfa de Cronbach que estudios similares como el de Lluch et al. (2013), realizado con una muestra de personas con trastornos crónicos de la salud física que obtuvo un alfa de 0.60, y el de Sequeira et al. (2014) con una muestra de estudiantes universitarios portugueses que obtuvo una puntuación de 0.51 en su alfa de Cronbach.

Si bien, según los resultados de la presente investigación no se logró la estructura original de la Escala de SMP, se observa que la conformación de los 6 factores mantiene la coherencia teórica del modelo subyacente, eso si, con una superposición de ítems en algunas de los factores como Satisfacción general que pasó de 8 ítems en la Escala de SMP original a 13 ítems en la versión colombiana y el factor Habilidades sociales que pasó de 7 ítems en la escala original a 4 ítems en la versión colombiana.

Por lo tanto, se sugiere adelantar al menos un nuevo estudio que realice análisis de confiabilidad con el estadístico Omega, que permite discriminar la estabilidad de las preguntas a partir de las cargas factoriales sin que dependa del número total de ítems (Gadermann, Guhn, \& Zumbo, 2004; Ventura-León \& Caycho-Rodríguez, 2017), lo cual podría reducir el posible sesgo que manifiesta el Alfa de Chronbach. Por otro lado, se aconseja aplicar otros métodos estadísticos de evaluación como la validez discriminante (Martínez-García \& Martínez-Caro, 2008), un método de análisis factorial confirmatorio con ecuaciones estructurales (Guardía-Olmos, 2016), o un análisis con Teoría de respuesta al Ítem (Attorresi, Lozzia, Abal, Galibert, \& Aguerri, 2009), que contrasten el modelo obtenido con valores de ajuste que permitan conclusiones más robustas. Esto con el fin de afinar el instrumento y hacerlo más preciso en la medición del constructo SMP en la población señalada.

La comparación con la versión original (española) del instrumento, como lo sugieren Sequeira et al. (2014), requerirá estudios adicionales en la versión colombiana para aclarar algunas dudas sobre la estructura de la Escala de SMP. En futuras investigaciones deberían realizarse análisis factoriales de confirmación y análisis de covarianza con muestras de mayor tamaño, a fin de seguir explorando tanto la estructura factorial del SMP como el Modelo Multifactorial de Salud Mental Positiva que lo sustenta.

La prueba analizada puede ser útil para la medición de los factores de Satisfacción General, Actitud Prosocial, Autocontrol, Autonomía y Solución de problemas - autoactualización, y en menor medida Habilidades sociales, pero se debe considerar además la correlación que tendrían cada uno de estos componentes con otras variables 
psicosociales como personalidad, regulación emocional, cognición social y estilos de afrontamiento, con el fin de obtener perfiles psicosociales completos de la población juvenil, y de acuerdo con ellos, ajustar las acciones en política pública en salud mental para este tipo de población (Gómez-Acosta, 2017).

Como limitación del presente estudio se indica que, si bien el tamaño muestral es suficiente y amplio para llevar a cabo los análisis realizados, dicha muestra no es aleatoria, por lo cual se sugiere replicar esta investigación con tamaños muestrales mayores.

\section{Agradecimientos}

Se agradece a las instituciones vinculantes de los investigadores por los tiempos dispuestos para el desarrollo del estudio.

\section{Conflicto de Intereses}

Los autores declaran la no existencia de conflicto de intereses.

\section{Referencias}

Amar, J., Palacio, J., Llinás, H., Puerta, L., Sierra, E., Pérez, A., \& Velásquez, B. (2008). Calidad de vida y salud mental positiva en menores trabajadores de Toluviejo. Suma Psicológica, 15(2), 385-403. Recuperado de http://publicaciones.konradlorenz.edu.co/index.php/sumapsi/article/view/45/79

Attorresi, H., Lozzia, G., Abal, F., Galibert, M., \& Aguerri, M. (2009). Teoría de Respuesta al Ítem: Conceptos básicos y aplicaciones para la medición de constructos psicológicos. Revista Argentina de Clínica Psicológica 18(2), 179-188. Recuperado de http://www.cienciared.com.ar/ra/usr/35/825/racp xviii 2 pp179 188.pdf

Barrera, M., \& Flores, M. (2013). Construcción de una escala de Salud Mental Positiva para Adultos en Población Mexicana. Revista Iberoamericana de Diagnóstico y Evaluación RIDEP, 39(1) 22-33. Recuperado de https://www.aidep.org/sites/default/files/articles/R39/Art2.pdf

Fernández-Ríos, L., \& Vilarino, M. (2018). Historia, investigación y discurso de la Psicología Positiva: un abordaje crítico. Terapia Psicológica, 36(2), 123 - 133. Recuperado de https://scielo.conicyt.cl/pdf/terpsicol/v36n2/0718-4808-terpsicol-36-02-00123.pdf

Gadermann, A., Guhn, M., \& Zumbo, B. (2012). Estimating ordinal reliability for Likerttype and ordinal item response data: A conceptual, empirical, and practical Guide. Practical Assessment, Research \& Evaluation, 17, 1-13. https://pareonline. net/pdf/v17n3.pdf

Ganga, N. S., \& Kutty, V. R. (2013). Influence of religion, religiosity and spirituality on positive mental health of young people. Mental Health, Religion \& Culture, 16(4), 435-443. doi: http://doi.org/10.1080/13674676.2012.697879

Gilmour, H. (2014). Positive mental health and mental illness. Health Reports, 25(9), 3-9. Recuperado de https://www150.statcan.gc.ca/n1/en/pub/82-003-x/2014009/ article/14086-eng.pdf?st=0VLzjN u

Gómez-Acosta, A. (2017). Predictores psicológicos del autocuidado en salud. Hacia la Promoción de la Salud, 22(1) 101-112, doi: http://doi.org/10.17151/ hpsal.2017.22.1.8

Gómez-Acosta, A., \& Londoño, C. (2013). Modelo predictor del consumo responsable de alcohol y el comportamiento típicamente no violento en adolescentes. Health \& Addictions Journal, 13(1), 23-34. Recuperado de https://www.redalyc.org/articulo. oa? id $=83928046003$ 
González, N., \& Valdés, J. (2016). Validez de la escala de Salud Mental Positiva en Niños Mexicanos. Acta de Investigación Psicológica, 6(1), 2368-2383. Recuperado de http://www.scielo.org.mx/pdf/aip/v6n1/2007-4719-aip-6-01-2368.pdf

González, P., Astudillo, E., \& Hurtado, E. (2012). Salud mental positiva y apoyo social percibido en personas con discapacidad física. Revista Cultura del Cuidado, 9(2), 3949. Recuperado de https://dialnet.unirioja.es/servlet/articulo?codigo $=6003024$

Guardía-Olmos, J. (2016). Esquema y recomendaciones para el uso de los Modelos de Ecuaciones Estructurales. Revista de Estudios e Investigación en Psicología y Educación, 3(2), 75-80. doi: http://doi.org/10.17979/reipe.2016.3.2.1847

Hurtado, B., Llunch-Canut, MT., Casas, I., Sequeira, C., Puig, M., \& Roldan, J. (2018). Evaluación de la fiabilidad y validez del cuestionario de salud mental positiva en profesores universitarios de enfermería en Cataluña. Revista de Enfermería y Salud Mental, 9, 5-17. Recuperado de https://dialnet.unirioja.es/servlet/articulo?codigo $=6258136$

Jahoda, M. (1958). Current Concept in Positive Mental Health. New York: Basic Book's.

Keyes, C., Dhingra, S., \& Simoes, E. (2010). Change in level of positive mental health as a predictor of future risk of mental illness. American Journal of Mental Health, 100(2), 2366-2371. https://ajph.aphapublications.org/doi/10.2105/ AJPH.2010.192245

Londoño, C. (2009). Optimismo y salud positiva como predictores de la adaptación a la vida universitaria. Acta Colombiana de Psicología, 12(1) 95-107. Recuperado de http://www.scielo.org.co/pdf/acp/v12n1/v12n1a09.pdf

Londoño, C., \& Alejo, I. (2017). Instrumentos usados en Colombia para evaluar la dimensión psicológica del proceso salud - enfermedad. Bogotá, D.C. Xpress Estudio Gráfico \& Digital S.A. Recuperado de https://publicaciones.ucatolica.edu.co/pdf/instrumentos-usados-en-colombia-para-evaluar-la-dimension-psicologica-del-proceso-salud-enfermedad.pdf

Lluch-Canut, T. (1999). Construcción de una escala para evaluar la salud mental positiva (tesis doctoral). Departamento de metodología de las ciencias del comportamiento. Barcelona. Universitat de Barcelona. Recuperado de http://diposit. ub.edu/dspace/bitstream/2445/42359/1/E_TESIS.pdf

Lluch-Canut, T. (2002). Evaluación empírica de un modelo conceptual de salud mental positiva. Salud Mental, 25(4), 42-55. Recuperado de http://www.revistasaludmental.mx/index.php/salud mental/article/view/914/912

Lluch-Canut T., Puig-Llobet M., Sanchez-Ortega A., Roldán-Merino, J., Ferré-Grau., \& Positive Mental Health Research Group. (2013). Assessing positive mental health in people with chronic physical health problems: correlations with socio-demographic variables and physical health status. BMC Public Health, 13, 928, doi: https://doi.org/10.1186/1471-2458-13-928

Martínez, R., Pando, M., León, S., González, R., Aldrete, M., \& López, L. (2015). Validez y confiabilidad de la escala de salud mental positiva. Psicogente, 18(33), 78-88. doi: http://doi.org/10.17081/psico.18.33.57

Martínez-García, J., \& Martínez-Caro, L. (2008). La validez discriminante como criterio de evaluación de escalas: ¿teoría o estadística? Universitas Psychologica 8(1), 27-36. Recuperado de https://revistas.javeriana.edu.co/index.php/revPsycho/ article/view/224

Medina, O., Serrano, M., Martínez, G., \& Cardona, D. (2012). Características de salud mental positiva en estudiantes de una universidad privada de la ciudad de Armenia-Quindío. Revista Virtual de la Universidad Católica del Norte, 1(36), 247-61. Recuperado de https://revistavirtual.ucn.edu.co/index.php/RevistaUCN/article/ view/379/714 
Muñoz. C., Restrepo, D., \& Cardona, D. (2016). Construcción del concepto de salud mental positiva: Revisión Sistemática. Revista Panamericana de Salud Pública, 39(3), 166-73. Recuperado de https://www.scielosp.org/article/ssm/content/raw/?resource ssm path=/media/assets/rpsp/v39n3/1020-4989-RPSP-39-03-166.pdf

Orellana, O., García, L., Yanac, E., Rivera, J., Alvites, J., \& Orellana, D. (2012). Competencias comunicativas y salud mental positiva en el perfil profesional de los profesores de la educación básica de Lima Metropolitana. Revista de Investigación en Psicología, 15(2), 61-87. doi: https://doi.org/10.15381/rinvp.v15i2.3688

Organización Mundial de la Salud [OMS]. (2018). Salud Mental: fortalecer nuestra respuesta. Recuperado de http://www.who.int/es/news-room/fact-sheets/detail/mental-health-strengthening-our-response

Salanova, M. (2008). Organizaciones saludables: una aproximación desde la psicología positiva. En C. Vázquez, \& y G. Hervás (Ed.), Psicología Positiva Aplicada (pp. 403-428). Bilbao: Desclee de Brower.

Seligman, M., \& Csikszentmihalyi, M. (2000). Positive Psychology: An Introduction. American Psychologist. 55, 5-14. doi: https://doi.org/10.1037/0003-066X.55.1.5

Sequiera, C., Carvalho, J., Sampaio, F., Sa, L., Lluch-Canut, T., \& Roldán-Merino, J. (2014). Evaluación de las propiedades psicométricas del Cuestionario de Salud Mental Positiva en estudiantes portugueses de educación superior. Revista Portuguesa de Enfermagem de Saúde Mental, 11, 45-53. Recuperado de http://www. scielo.mec.pt/pdf/rpesm/n11/n11a07.pdf

Srivastava, K. (2011). Positive mental health and its relationship with resilience. Industrial Psychiatry Journal, 2(2), 75-76. doi: https://doi.org/10.4103/0972$\underline{6748.102469}$

Vaillant, G. (2012). Positive mental health: is there a cross-cultural definition? World Psychiatry, 11(2), 93-9. Recuperado de https://www.ncbi.nlm.nih.gov/pmc/articles/PMC3363378/pdf/wpa020093.pdf

Vázquez, C., Hervas, G., Rahona, J., \& Gómez, D. (2009). Bienestar Psicológico y Salud: Aportaciones desde la Psicología Positiva. Anuario de la Psicología Clínica y de la Salud, 5, 15-28. Recuperado de http://institucional.us.es/apcs/doc/APCS 5 esp 15-28.pdf

Ventura-León, J., \& Caycho-Rodríguez, T. (2017). El coeficiente Omega: un método alternativo para la estimación de la confiabilidad. Revista Latinoamericana de Ciencias Sociales, Niñez y Juventud, 15(1), 625-627. Recuperado de https://www. redalyc.org/pdf/773/77349627039.pdf

Vidal, D., Rivas, E., \& Bustos, L. (2015). Nivel de salud mental positiva en equipos de salud mental de tres hospitales de Chile, 2013. Cuidados de Enfermería y Educación en Salud, 2(1), 48-62. Recuperado de https://revistas.userena.c//index.php/ cuidenf/article/view/647/767 